\title{
Investigation of Sphere Decoder and Channel Tracking Algorithms for Media-Based Modulation over Time-Selective Channels
}

\author{
Yongzhi Li, ${ }^{1}$ Cheng Tao, ${ }^{1,2}$ Yapeng Li, ${ }^{1}$ Liu Liu, ${ }^{1,2}$ and Tao Zhou ${ }^{1}$ \\ ${ }^{1}$ Institute of Broadband Wireless Mobile Communications, Beijing Jiaotong University, Beijing, China \\ ${ }^{2}$ National Mobile Communications Research Laboratory, Southeast University, Nanjing, China \\ Correspondence should be addressed to Cheng Tao; chtao@bjtu.edu.cn
}

Received 5 April 2017; Revised 15 July 2017; Accepted 9 August 2017; Published 17 September 2017

Academic Editor: Gonzalo Vazquez-Vilar

Copyright (C) 2017 Yongzhi Li et al. This is an open access article distributed under the Creative Commons Attribution License, which permits unrestricted use, distribution, and reproduction in any medium, provided the original work is properly cited.

\begin{abstract}
The performance of media-based modulation (MBM) systems, where additional information can be conveyed by the indices of the channel states created by RF mirrors, over time-selective channels is investigated. By transforming the MBM system model into a traditional MIMO system model, we first propose a reduced complexity sphere decoder algorithm. Then two channel tracking algorithms, which are based on least mean square adaptive filter and recursive least-squares adaptive filter, are employed in order to combat the performance loss caused by the time-varying channels. Numerical results show that the proposed sphere decoder and these two channel tracking algorithms perform well in MBM systems.
\end{abstract}

\section{Introduction}

Recently, media-based modulation (MBM) [1-3] has been proposed as a new technology to improve the spectral efficiency. Compared to the traditional modulation, where the information is embedded in the RF source (namely, the source-based modulation (SBM) as referred in [1]), additional information can be embedded into the indices of the active channel states in MBM systems. This can be achieved, for example, by placing a number of mirrors around each transmit antenna and indexing the mirror configuration.

MBM can be regarded as an instance of index modulation (or channel modulation as described in [4]), which has attracted significant attention recently due to the advantages in significantly improving the spectral and energy efficiency compared to the traditional digital modulation technique. For index modulation techniques, additional information bits can be embedded into the indices of the building blocks of the considered communication systems [5]. Although the concepts of antenna selection technique $[6,7]$ and reconfigurable antennas $[8,9]$ are very similar to the concept of index modulation, the main difference is that the antenna selection and reconfigurable antennas techniques do not index the activated antennas indices and the antenna patterns to convey additional information bits. One application of index modulation is the space shift keying (SSK) [10]. In SSK, the transmitter only activates one out of all the transmit antennas to convey the information bit, and hence the spectral efficiency scales only logarithmically in the number of transmit antennas. This implies that the SSK should exponentially increase the number of transmit antennas in order to linearly improve the spectral efficiency, which will also exponentially increase the system hardware cost in terms of both economic and energy consumption. However, these drawbacks for SSK can be significantly reduced in the MBM system, since the spectral efficiency in MBM only scales linearly with the number of RF mirrors, while the number of transmit antennas remains fixed.

For MBM technique, it has been proved in [2] that a $1 \times N$ MBM over static multipath channel asymptotically achieves the capacity of $N$ parallel AWGN channels, where for each unit of energy over the single transmit antenna, the effective energy for each AWGN channel is the statistical average of channel fading. It is confirmed in [3] that the performances of SIMO-MBM are better than the SBM in some aspects and a significant coding gain can be obtained if 


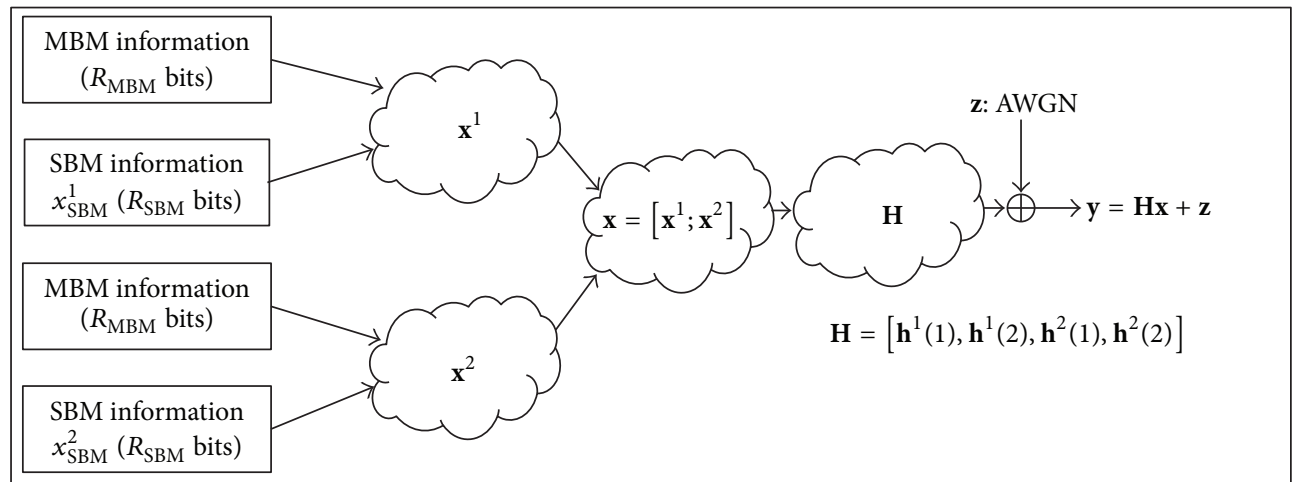

Figure 1: Equivalent system model for MIMO-MBM with $U=2$ transmit units.

single parity check symbol code is applied to a SIMO-MBM. The authors in [11] presented the layered MIMO mediabased modulation (LMIMO-MBM), which overcomes the recovery complexity issues at the cost of losing symbol error rate performance. By combining the space-time block coding and MBM, the concept of space-time channel modulation scheme is introduced in [4]. It is shown in [4] that proposed schemes achieve considerably better error performance than the existing MBM systems. Reference [12] combined MBM and space modulation technique to further improve the error performance of MIMO-MBM systems. More importantly, a simple and convenient model, which is named generalized spatial modulation-MBM (GSM-MBM), is proposed in [12]. Based on [12], the intricate MBM model originally described in [1] can be reformed as an explicit MIMO system model, which allows us to use existing MIMO algorithm to handle the newly raised problem in MBM systems.

There have been a variety of existing works on the performance of MBM systems. However, the performance of MBM systems over time-selective channels has not been previously treated. Thus, in this paper, we investigate the performance of the MBM systems over time-selected channels by using the GSM-MBM model. Based on this model, traditional MIMO decoding algorithms can be used in MBM systems. Although the maximum likelihood (ML) decoder is optimal, the algorithm complexity is prohibited in practice. While the authors in [3] proposed a novel detection algorithm for SIMO-MBM based on sphere decoder to improve the processing speed of the receiver, we note that there is still room to reduce the complexity of sphere decoder. Therefore, in our work, we first present a reduced complexity sphere decoder for the MIMO-MBM systems. As illustrated later, our proposed decoder has almost the same performance as ML decoder. Then the symbol error rate (SER) performance of MIMO-MBM over time-selective channels is investigated using the proposed decoder method and two tracking algorithms called least mean square- (LMS-) MBM and recursive least-squares- (RLS-) MBM are proposed to improve the performance of MIMO-MBM. Numerical simulations verify that the proposed channel tracking algorithms can perform well in MBM systems.

\section{System Model}

In this section, we consider a $U \times N$ MIMO system used with MBM technique and assume that there are $U R_{\mathrm{MBM}}+U R_{\mathrm{SBM}}$ bits information to be transmitted per channel use, where $R_{\mathrm{MBM}}$ bits are the MBM information, $R_{\mathrm{SBM}}$ bits are the SBM information, and $U$ and $N$ represent the number of transmit and receive antennas, respectively. In such system, each transmit unit can create $M=2^{R_{\mathrm{MBM}}}$ independent channel states by turning on/off $R_{\mathrm{MBM}}$ RF mirrors. By employing the GSM-MIMO model described in [12], the received signal at the receiver can be expressed as

$$
\mathbf{y}=\mathbf{H x}+\mathbf{z}
$$

where $\mathbf{H} \in \mathbb{C}^{N \times U M}$ and $\mathbf{x} \in \mathbb{C}^{U M \times 1}$ represent the overall channel matrix and the overall data symbol vector, respectively. More precisely, the overall channel matrix and the overall data symbol vector are given by

$$
\begin{aligned}
\mathbf{x} & =\left[\mathbf{x}^{1}, \ldots, \mathbf{x}^{u}, \ldots, \mathbf{x}^{U}\right], \\
\mathbf{H} & =\left[\mathbf{H}^{1}, \ldots, \mathbf{H}^{u}, \ldots, \mathbf{H}^{U}\right],
\end{aligned}
$$

where $\mathbf{x}^{u}=\left[0, \ldots, 0, x_{\mathrm{SBM}}^{u}, 0, \ldots, 0\right]^{T}$ with $x_{\mathrm{SBM}}^{u}$ denoting the data symbol modulated by $R_{\mathrm{SBM}}$ bits and $\mathbf{H}^{u}=$ $\left[\mathbf{h}^{u}(1), \mathbf{h}^{u}(2), \ldots, \mathbf{h}^{u}(M)\right]$ with $\mathbf{h}^{u}(m) \in \mathbb{C}^{N \times 1}$ denoting the $m$ th channel state at the $u$ th transmit antenna, respectively. $\mathbf{z} \sim \mathscr{C} \mathscr{N}\left(\mathbf{0}, \sigma^{2} \mathbf{I}\right)$ is the Gaussian noise, where we assume that each element has zero-mean and finite variance $\sigma^{2}$. Note that the system model in (1) is a special case of the GSMMBM model by assuming that all the transmit antennas are activated. Thus, in the following, we refer to the system model in (1) as MIMO-MBM.

Next, we will take an example to illustrate how to use the equivalent system model to realize the process of MIMOMBM. For simplicity, we take the example as illustrated in Figure 1, where a MIMO-MBM system with $U=2, N=4$, $R_{\mathrm{MBM}}=1$, and $R_{\mathrm{SBM}}=1$ is considered. According to (1)-(2), the received signal can be expressed as

$$
\mathbf{y}=\left[\mathbf{h}^{1}(1), \mathbf{h}^{1}(2), \mathbf{h}^{2}(1), \mathbf{h}^{2}(2)\right] \times\left[\mathbf{x}^{1} ; \mathbf{x}^{2}\right]+\mathbf{z} .
$$


TABLE 1: MIMO-MBM mapping process.

\begin{tabular}{lccc}
\hline$R_{\mathrm{MBM}}$ bits & $\mathbf{h}^{u}(m)$ & $\mathbf{x}_{\mathrm{MBM}}^{u}$ & $\mathbf{x}^{u}$ \\
\hline 0 & $\mathbf{h}^{u}(1)$ & {$[1,0]^{T}$} & {$\left[x_{\mathrm{SBM}}^{u}, 0\right]^{T}$} \\
1 & $\mathbf{h}^{u}(2)$ & {$[0,1]^{T}$} & {$\left[0, x_{\mathrm{SBM}}^{u}\right]^{T}$} \\
\hline
\end{tabular}

Then different transmit units can send data symbol using the rule in Table 1 . If the $u$ th transmit unit sends MBM information 0 and SBM information $x_{S B M}^{u}$, which implies that the $u$ th channel $\mathbf{h}^{u}(1)$ is used to convey the SBM data symbol $x_{\mathrm{SBM}}^{u}$, then the whole transmit data of the $u$ th transmit unit can be represented as $\mathbf{x}^{u}=\left[x_{\mathrm{SBM}}^{u}, 0\right]$. If the $u$ th transmit unit sends MBM information 1 and SBM information $x_{\text {SBM }}^{u}$, which implies that the $u$ th channel $\mathbf{h}^{u}(2)$ is used to convey the SBM data symbol $x_{\text {SBM }}^{u}$, then the whole transmit data of the $u$ th transmit unit can be represented as $\mathbf{x}^{u}=\left[0, x_{\mathrm{SBM}}^{u}\right]$.

Intuitively, if the receiver can perfectly recover the transmit data $\left[\mathbf{x}^{1} ; \mathbf{x}^{2}\right]$, then the MBM information $\left[\mathbf{x}_{\mathrm{MBM}}^{1} ; \mathbf{x}_{\mathrm{MBM}}^{2}\right]$ can be obtained by using the same rule as shown in Table 1. In this way, both the MBM information and SBM information are demodulated at the receiver.

\section{Reduced Complexity Sphere Decoder for MIMO-MBM}

By using the generalized system model in (1), the receiver can recover the transmit data symbol $\mathbf{x}$ using existing decoder methods, that is, the ML decoder. However, the complexity of ML decoder is very high, especially when the constellation size is large. For completeness, we provide the computational complexity of ML decoder in the sequel.

At the receiver side, the ML decoder is considered to detect the signal $\mathbf{x}$ as

$$
\widehat{\mathbf{x}}=\underset{\mathbf{x} \in \mathscr{A}}{\operatorname{argmin}}\|\mathbf{y}-\mathbf{H} \mathbf{x}\|^{2}
$$

where $\mathscr{A}$ is the set of all possible x's. We then evaluate the complexity of ML decoder using the number of add/ multiply/comparisons operations [3]:

$$
\psi_{\mathrm{ML}}=\psi_{\mathrm{ML} \_ \text {add }}+\psi_{\mathrm{ML} \_ \text {multiply }}+\psi_{\mathrm{ML} \_ \text {comparison }}
$$

where $\psi_{\text {ML_add }}=(4 U N+4 N-1) M^{U} \widehat{M}, \psi_{\text {ML_multiply }}=4 N(U+$ 1) $M^{U} \widehat{M}, \psi_{\text {ML_comparison }}=M^{U} \widehat{M}, \widehat{M}$ is the constellation size, and $N$ is the number of receive antennas. Here we take the quadrature amplitude modulation (QAM) for example. We can easily see that the complexity of ML decoder is prohibitive if the whole constellation size of MBM is large. Nevertheless, we note that the complexity of comparison operation is relatively lower than that of add/multiply operation. Therefore, we aim at reducing the number of add/multiply operations at the cost of increasing a few more comparison operations in order to reduce the complexity of the recovery process. In particular, we will propose a modified sphere decoder (SD) for MBM to achieve the same performance as the ML decoder.
The considered SD for MBM, which is called SD-MBM algorithm later in this paper, is a modified version of the SD algorithm presented in [13]. Assume that $r$ is the sphere radius and the SD can be expressed as

$$
\widehat{\mathbf{x}}=\underset{\mathbf{x} \in \mathscr{A}}{\operatorname{argmin}}\left(\|\mathbf{y}-\mathbf{H} \mathbf{x}\|^{2} \leq r^{2}\right),
$$

which means that SD performs a ML search only through those points in the sphere with radius $r$. If $r$ is sufficiently large, then the solution of (6) will be the same as the solution of (4). If a candidate $\mathbf{x}_{a}$ is within the search radius $r$, then the following equation must be true:

$$
\sum_{j=1}^{n}\left(y_{j}-\mathbf{h}_{j} \mathbf{x}_{a}\right)^{2} \leq r^{2} \quad \forall 1 \leq n \leq N,
$$

where $\mathbf{h}_{j}$ is the $j$ th row of $\mathbf{H}$. In other words, a candidate can be rejected from further calculation if any partial sums are out of the sphere. This property reduces the amount of work required to evaluate candidates when the constellation size is large.

In fact, if we find a point in the sphere, we can update the radius with the Euclidean distance of that point to further reduce the complexity. But the initial sphere radius is not easy to find because if the radius is too large, there will be too many points to be searched; if the radius is too small, there will be no point to be found. The authors in [7] give a conventional method of deciding initial radius:

$$
r^{2}=\zeta N \sigma^{2}
$$

where $\sigma^{2}$ is the variance of noise, $N$ is the number of receive antennas, and $\zeta$ is a constant number based on experience.

If we set the radius $r$ properly, we can always find the optimal solution and reduce the complexity. If we set the radius small occasionally, no point is in the sphere after the search and hence we need to enlarge the radius and do the search again. In this case, fortunately, since many points have been searched last time, we do not need to start over; we only need to start from where we stopped at last time and hence each point is searched no more than once. In this way, the numbers of add and multiply operations are reduced only at the cost of a few more comparisons. In what follows, we provide the full procedure of SD-MIMO-MBM: let $C$ be the constellation size of $\mathrm{MBM}$, let $\mathscr{A}$ be a set containing all possible transmit signals, $\mathscr{A}=\left\{\mathbf{x}_{1}, \mathbf{x}_{2}, \ldots, \mathbf{x}_{C}\right\}$, let $l(i)$ be the position of search along path $i$, and let $d\left(\mathbf{x}_{i}\right)$ be the Euclidean distance along path $i$. Then the algorithm can be formulated as Algorithm 1 .

The advantage of the modified SD-MIMO-MBM is that it always can find a point in the sphere and search every point along each path no more than once, which reduces the complexity of the decoding process significantly.

\section{Time-Selective Channels Model for MIMO-MBM}

It is known that a moving terminal, especially for the terminal on the high-speed railway [14], will induce large Doppler 
(1) $l(i)=1, i=1,2, \ldots, C, d\left(\mathbf{x}_{i}\right)=0, i=1,2, \ldots, C$;

(2) for $i_{1}=1: C$ do

(3) for $i_{2}=l\left(i_{1}\right): N$ do

(4) if $d\left(\mathbf{x}_{i_{1}}\right)>r^{2}$ then

(5) $\quad l\left(i_{1}\right)=i_{2}$ and go to $(10)$

(6) end if

(7) $\quad d\left(\mathbf{x}_{i_{1}}\right)+=\left|\mathbf{y}\left(i_{2}\right)-\mathbf{H}\left(i_{2},:\right) \mathbf{x}_{i_{1}}\right|^{2}$;

(8) $\quad l\left(i_{1}\right)=i_{2}+1$;

(9) end for

(10) if $d\left(\mathbf{x}_{i_{1}}\right)<r^{2}$ then

(11) $r^{2}=d\left(\mathbf{x}_{i_{1}}\right)$;

(12) end if

(13) end for

(14) if $\left\{d\left(\mathbf{x}_{i}\right)>r^{2}\right.$ for $\left.\forall i \in\{1,2, \ldots, C\}\right\} \&\{l(i)<N+1$ for $\exists i \in\{1,2, \ldots, C\}\}$ then

(15) $r^{2}=1.2 r^{2}$ and go to (2)

(16) end if

(17) $\widehat{\mathbf{x}}=\operatorname{argmin}_{i \in\{1,2, \ldots, C\}} d\left(x_{i}\right)$

(18) return $\widehat{\mathbf{x}}$

Algorithm 1: Modified SD algorithm for MIMO-MBM.

shift effect, which is the cause of the time-selectivity in the fast-fading channel. Therefore, in this section, we use an improved sum-of-sinusoids statistical simulation model of Rayleigh fading channels to model time-selective channels property and study the performance of MIMO-MBM. The normalized low-pass fading process of a new statistical sumof-sinusoids simulation model is defined by [15]

$$
\begin{aligned}
h[k] & =h_{I}[k]+j h_{\mathrm{Q}}[k], \\
h_{I}[k] & =\sqrt{\frac{2}{W}} \sum_{w=1}^{W} \cos \left(\omega_{d} k \cos \beta_{w}+\phi_{w}\right), \\
h_{\mathrm{Q}}[k] & =\sqrt{\frac{2}{W}} \sum_{w=1}^{W} \cos \left(\omega_{d} k \sin \beta_{w}+\varphi_{w}\right),
\end{aligned}
$$

where $\beta_{w}=(2 \pi w-\pi+\theta) / 4 W, w=1,2, \ldots, W, \phi_{w}, \varphi_{w}$, and $\theta$ are statistically independent and uniformly distributed in the range of $[-\pi, \pi)$ for all $w$, and $W$ is the number of sinusoids. Thus, the channel matrix of MIMO-MBM at time instant $k$ can be represented as

$$
\mathbf{H}[k]=\left[\begin{array}{cccc}
h_{1}^{1}[1, k] & h_{1}^{1}[2, k] & \ldots & h_{1}^{U}[M, k] \\
h_{2}^{1}[1, k] & h_{2}^{1}[2, k] & \ldots & h_{2}^{U}[M, k] \\
h_{3}^{1}[1, k] & h_{3}^{1}[2, k] & \ldots & h_{3}^{U}[M, k] \\
\vdots & \vdots & h_{n}^{u}[m, k] & \vdots \\
h_{N}^{1}[1, k] & h_{N}^{1}[2, k] & \ldots & h_{N}^{U}[M, k]
\end{array}\right],
$$

where $h_{n}^{u}[m, k]$ denotes the $m$ th channel coefficient between the $u$ th transmit unit and the $n$th receive antenna at $k$ th time instant and is described as (9). We use autocorrelation to model the variation of channel factors with time [16]:

$$
\alpha=E\left\{h_{n}^{u}\left[m, k_{1}\right] h_{n}^{u}\left[m, k_{2}\right]^{*}\right\}=J_{0}\left(\omega_{d}\left(k_{1}-k_{2}\right)\right),
$$

where $J_{0}(\cdot)$ is the 0th-order Bessel function of the first kind and $\omega_{d}$ is the maximum Doppler shift. The coefficient $\alpha$ relies on $\omega_{d}$ and $k_{1}-k_{2}$.

\section{Channel Tracking for MIMO-MBM}

In this section, we discuss how to obtain the channel matrix at the receiver and improve the performance of the decoding process. During the channel estimation phase, the transmitter sends pilots by scanning through the whole constellation space. The accuracy of estimated channel matrix $\widehat{\mathbf{H}}$ depends on the length of pilots $T_{t}$ [17]. The initial estimated channel matrix can be expressed as [18]

$$
\widehat{\mathbf{H}}=\sqrt{1-t^{2}} \mathbf{H}+t \widetilde{\mathbf{H}},
$$

where $\mathbf{H}$ is the exact channel matrix, $\widetilde{\mathbf{H}}$ is the estimation error matrix, and $t \in[0,1]$ is the scale of estimation error. The estimated channel matrix $\widehat{\mathbf{H}}$ is used to recover the data at the receiver. Intuitively, for time-selective channels, the symbol error rate will be larger than that in time-invariant channel. Therefore, it is important to use channel tracking to update channel matrix in order to improve the system performance.

5.1. Least Mean Square (LMS) Algorithm for MIMO-MBM. The adaptive LMS algorithm is widely used in practice due to its simplicity, computational efficiency, and good performance under a variety of operating conditions. It is based on minimum mean squared error (MMSE) criterion and is introduced in [19] in the form of vectors updating problem. We use it to track the channel matrix of MIMO-MBM in time-selective channels and develop an algorithm to improve the performance of recovery process at the receiver side. We assume that the channel matrix $\widehat{\mathbf{H}}[k]$ is obtained after the pilot training at time instant $k$. We also assume that the receiver employs the channel estimate $\widehat{\mathbf{H}}[k]$ to detect the 
transmit data symbol $\widehat{\mathbf{x}}[k+1]$ at time $k+1$. Then the estimation error vector is

$$
\mathbf{e}[k+1]=\mathbf{y}[k+1]-\widehat{\mathbf{H}}[k] \widehat{\mathbf{x}}[k+1],
$$

where $\mathbf{y}[k+1]$ is the received signal at time $k+1$. So the mean squared error (MSE) can be expressed as

$$
\xi=E\left\{\|\mathbf{e}[k+1]\|^{2}\right\}=E\left\{\mathbf{e}[k+1]^{H} \mathbf{e}[k+1]\right\} .
$$

Then, substituting (15) into (16) and calculating the derivative of (16) yield

$$
\nabla_{\widehat{\mathbf{H}}} \xi=E\left\{2(\widehat{\mathbf{H}}(t) \widehat{\mathbf{x}}[k+1]-\mathbf{y}[k+1]) \widehat{\mathbf{x}}^{H}[k+1]\right\} .
$$

Since our goal is to minimize MSE of (16), we update the channel matrix using (18):

$$
\begin{aligned}
\widehat{\mathbf{H}}[k+1] & =\widehat{\mathbf{H}}[k]+\mu\left(-\nabla_{\widehat{\mathbf{H}}} \xi\right) \\
& =\widehat{\mathbf{H}}[k]+2 \mu \mathbf{e}[k+1] \widehat{\mathbf{x}}^{H}[k+1],
\end{aligned}
$$

where $\mu$ is the step size. According to [19], in order to simplify the selection of step size $\mu$, we employ the normalized LMSMBM algorithm to update the channel matrix:

$$
\widehat{\mathbf{H}}[k+1]=\widehat{\mathbf{H}}[k]+\frac{\mu}{E_{\widehat{\mathbf{x}}}[k+1]} \mathbf{e}[k+1] \widehat{\mathbf{x}}^{H}[k+1],
$$

where $E_{\widehat{\mathbf{x}}}[k+1]=\|\widehat{\mathbf{x}}[k+1]\|^{2}$ and $0<\mu<1$. After obtaining the channel matrix $\widehat{\mathbf{H}}[k+1]$, the receiver recalculates $\widehat{\mathbf{x}}[k+1]$ with $\widehat{\mathbf{H}}[k+1]$ and $\mathbf{y}[k+1]$ as the decoded signal.

The idea of LMS-MBM algorithm is that the receiver updates the estimated channel matrix to decode signal and reduce MSE. The implementation of the adaptive algorithm starts with a training transmission, which is used to acquire initial $\widehat{\mathbf{H}}\left[k_{0}\right]$ estimates. Then we use LMS-MBM algorithm to estimate channel matrix and detect the transmit data symbol. The steps of LMS-MBM algorithm are summarized as follows:

Step 1: initial $\widehat{\mathbf{H}}\left[k_{0}\right], \mu$, and parameter $\tau=1$

Step 2: recovering $\widehat{\mathbf{x}}\left[k_{0}+\tau\right]$ with estimated channel matrix $\widehat{\mathbf{H}}\left[k_{0}+\tau-1\right]$ and received signal $\mathbf{y}\left[k_{0}+\tau\right]$ : $\mathcal{O}\left(N U M^{U+1}\right)$

Step 3: calculating estimation error $\mathbf{e}\left[k_{0}+\tau\right]$ : O(NUM)

Step 4: updating the channel matrix $\widehat{\mathbf{H}}\left[k_{0}+\tau\right]$ : $\mathcal{O}(N U M)$

Step 5: recalculating $\widehat{\mathbf{x}}\left[k_{0}+\tau\right]$ with the updated channel matrix $\widehat{\mathbf{H}}\left[k_{0}+\tau\right]$; then $\tau=\tau+1$ and go to Step 2: $\mathcal{O}\left(N U M^{U+1}\right)$

$\widehat{\mathbf{x}}[k], \mathbf{y}[k]$, and $\widehat{\mathbf{H}}[k]$ are the estimated symbol, the received signal, and the estimated channel matrix at time $k$, respectively. $\mu$ is the step size parameter of updating process and $0<\mu<1 ; \tau$ is the time of data transmission after pilot transmission. The calculation complexity is listed at the end of each step.
5.2. Recursive Least Square (RLS) Algorithm for MIMO-MBM. The complexity of RLS algorithm [19] is a little higher than that of LMS algorithm because it considers the minimization of the total weighted squared error, which depends on all received signals and all transmitted symbols before time instant $k$ and the channel matrix at time $k$. Therefore, the channel matrix at time index $k$ is chosen to minimize the cost function:

$$
e[k]=\sum_{j=1}^{k} \lambda^{k-j}\|\mathbf{y}[j]-\widehat{\mathbf{H}}[k] \widehat{\mathbf{x}}[j]\|^{2},
$$

where $\lambda$ is the forgetting factor and $0<\lambda<1$. We try to obtain channel matrix $\widehat{\mathbf{H}}[k]$ to reduce the weighted squared error and calculate the derivative of (20):

$$
\frac{\partial e[k]}{\partial \widehat{\mathbf{H}}[k]}=2 \widehat{\mathbf{H}}[k] \mathbf{R}[k]-2 \mathbf{D}[k]=0,
$$

where

$$
\begin{aligned}
& \mathbf{R}[k]=\sum_{j=1}^{k} \lambda^{k-j} \widehat{\mathbf{x}}[k] \widehat{\mathbf{x}}^{H}[k], \\
& \mathbf{D}[k]=\sum_{j=1}^{k} \lambda^{k-j} \mathbf{y}[j] \widehat{\mathbf{x}}^{H}[j] .
\end{aligned}
$$

From (22), we have

$$
\begin{aligned}
& \mathbf{R}[k]=\lambda \mathbf{R}[k-1]+\widehat{\mathbf{x}}[k] \widehat{\mathbf{x}}^{H}[k], \\
& \mathbf{D}[k]=\lambda \mathbf{D}[k-1]+\mathbf{y}[k] \widehat{\mathbf{x}}^{H}[k] .
\end{aligned}
$$

Substituting $\mathbf{R}[k-1]$ and $\mathbf{D}[k-1]$ into (21), we obtain

$$
\widehat{\mathbf{H}}[k-1] \mathbf{R}[k]+\mathbf{e}[k] \widehat{\mathbf{x}}^{H}[k]=\mathbf{D}[k],
$$

where

$$
\mathbf{e}[k]=\mathbf{y}[k]-\widehat{\mathbf{H}}[k-1] \widehat{\mathbf{x}}[k] .
$$

By assuming that $\mathbf{R}[k]$ is invertible and multiplying both sides of (24) by $\mathbf{R}^{-1}[k]$, we can obtain

$$
\begin{aligned}
\widehat{\mathbf{H}} & {[k-1]+\mathbf{e}[k] \widehat{\mathbf{x}}^{H}[k] \mathbf{R}^{-1}[k]=\mathbf{D}[k] \mathbf{R}^{-1}[k] } \\
& =\widehat{\mathbf{H}}[k] .
\end{aligned}
$$

The step size factor is defined as

$$
\mathbf{g}[k]=\widehat{\mathbf{x}}^{H}[k] \mathbf{R}^{-1}[k] .
$$

Similarly, substituting $\mathbf{R}[k]$ and $\mathbf{D}[k]$ into (21), we obtain

$$
\lambda \widehat{\mathbf{H}}[k] \mathbf{R}[k-1]-\varepsilon[k] \widehat{\mathbf{x}}^{H}[k]=\lambda \mathbf{D}[k-1],
$$

where

$$
\varepsilon[k]=\mathbf{y}[k]-\widehat{\mathbf{H}}[k] \widehat{\mathbf{x}}[k] .
$$


By assuming that $\mathbf{R}[k-1]$ is invertible and multiplying both sides of (28) by $\mathbf{R}^{-1}[k-1]$, we can obtain

$$
\begin{aligned}
\widehat{\mathbf{H}} & {[k]-\frac{1}{\lambda} \varepsilon[k] \widehat{\mathbf{x}}^{H}[k] \mathbf{R}^{-1}[k-1] } \\
& =\mathbf{D}[k-1] \mathbf{R}^{-1}[k-1]=\widehat{\mathbf{H}}[k-1] .
\end{aligned}
$$

The step size factor is defined as

$$
\overline{\mathbf{g}}[k]=\frac{1}{\lambda} \widehat{\mathbf{x}}^{H}[k] \mathbf{R}^{-1}[k-1] .
$$

Combining (30) with (29), we get

$$
\mathbf{e}[k]=\varepsilon[k] \bar{\alpha}[k],
$$

where

$$
\bar{\alpha}[k]=1+\overline{\mathbf{g}}[k] \mathbf{x}[k] .
$$

Similarly, combining (26), (30), and (32), we obtain

$$
\overline{\mathbf{g}}[k]=\bar{\alpha}[k] \mathbf{g}[k],
$$

which shows that the two adaptation gains have the same direction but different mold. And the major problem is how to update step size factor $\mathbf{g}[k]$ or $\overline{\mathbf{g}}[k]$. By defining

$$
\mathbf{P}[k]=\mathbf{R}^{-1}[k],
$$

we have such an updating

$$
\mathbf{P}[k]=\lambda^{-1} \mathbf{P}[k-1]-\mathbf{g}^{H}[k] \overline{\mathbf{g}}[k],
$$

which is proven in [19] and omitted here. The update of channel matrix can be achieved by using (27), (31), (33), (34), (35), (36), and (26).

We use this algorithm called RLS-MBM to track the channels of generalized media-based modulation and improve the performance of symbol error rate. It is summarized as follows:

Step 1: initial $\widehat{\mathbf{H}}\left[k_{0}\right], \mathbf{P}\left[k_{0}\right]$, parameter $\lambda$, and $\tau=1$

Step 2: recovering $\widehat{\mathbf{H}}\left[k_{0}+\tau-1\right]$ with received signal $\mathbf{y}\left[k_{0}+\tau\right]$ and estimated channel matrix $\widehat{\mathbf{H}}\left[k_{0}+\tau-1\right]$ : $\mathcal{O}\left(N U M^{U+1}\right)$

Step 3: calculating step size parameter $\overline{\mathbf{g}}\left[k_{0}+\tau\right], \bar{\alpha}\left[k_{0}+\right.$ $\tau]$, and $\mathbf{g}\left[k_{0}+\tau\right]: \mathcal{O}\left(U^{2} M^{2}\right)$

Step 4: calculating estimation error $\mathbf{e}\left[k_{0}+\tau\right]$ : $\mathcal{O}(N U M)$

Step 5: updating the channel matrix $\widehat{\mathbf{H}}\left[k_{0}+\tau\right]$ : $\mathcal{O}(N U M)$

Step 6: recalculating $\widehat{\mathbf{x}}\left[k_{0}+\tau\right]$ with the updated channel matrix $\widehat{\mathbf{H}}\left[k_{0}+\tau\right]$ and $\mathbf{y}\left[k_{0}+\tau\right]: \mathcal{O}\left(N U M^{U+1}\right)$

Step 7: updating $\mathbf{P}\left[k_{0}+\tau\right]$; then $\tau=\tau+1$ and go to Step 2: $\mathcal{O}\left(U^{2} M^{2}\right)$

$\widehat{\mathbf{x}}[k] \mathbf{y}[k]$ and $\widehat{\mathbf{H}}[k]$ are the estimated symbol, the received signal, and the estimated channel matrix at time $k$, respectively. $\lambda$ is the forgetting factor and $0<\lambda<1 ; \delta$ is a large constant initial parameter and $\tau$ is the data transmission time after pilot transmission.
5.3. Complexity Analysis for LMS-MBM and RLS-MBM. The aim of this subsection is to present the computational complexity of the proposed LMS-MBM and RLS-MBM channel tracking algorithms. The LMS-MBM and RLS-MBM algorithms are shown in Sections 5.1 and 5.2, respectively, with the complexity corresponding to each step of the algorithms. As we can see, the overall order of complexity of LMS-MBM is given by

$$
\psi_{\text {LMS-MBM }}=\mathcal{O}\left(N U M^{U+1}\right) .
$$

As for RLS-MBM, the overall order of complexity is given by

$$
\psi_{\text {RLS-MBM }}=\mathcal{O}\left(\max \left\{M U, N M^{U}\right\} U M\right) .
$$

In practice, we assume that $M \geq U$ because the SER performance loss of MIMO-MBM is huge when $U$ is large. Therefore, if $U \geq 2, \psi_{\text {RLS-MBM }}=\mathcal{O}\left(N U M^{U+1}\right)$, which means that the complexities of LMS-MBM and RLS-MBM are the same. If $U=1, \psi_{\text {RLS-MBM }}=\mathscr{O}\left(N M^{2}\right)$ and $\psi_{\text {LMS-MBM }}=\mathscr{O}\left(N M^{2}\right)$. Therefore, the computational complexities of LMS-MBM and RLS-MBM are equal.

\section{Numerical Simulations}

In this section, we present simulations to evaluate the performance of proposed sphere decoder for MIMO-MBM and the tracking ability of LMS-MBM and RLS-MBM for MIMOMBM in time-selective channels. The time-invariant channel vectors corresponding to each transmit unit are generated with complex i.i.d. Gaussian random components of unit variance, while the time-selective channel vectors of each transmit unit are generated according to Section 4. Energy per bit is defined as the sum of signal energies of transmit units divided by the total number of bits per channel use. Thus the signal-to-noise rate (SNR) is defined as [20]

$$
\mathrm{SNR}=\frac{U E_{U}}{R N_{0}},
$$

where $E_{U}$ is the signal energy of each transmit unit and $R$ is the number of bits per channel use. Other default values of various parameters are listed in Table 2. We employ average symbol error rate (SER) and mean square error (MSE) of channel estimation as performance metrics to evaluate performances of MIMO-MBM. MSE of channel estimation is defined as [21]

$$
\text { MSE }=\frac{1}{U N M} \sum_{i=1}^{N} \sum_{j=1}^{M} \sum_{u=1}^{U} E\left\{\left\|\widehat{\mathbf{h}}_{i}^{u}[j, k]-\mathbf{h}_{i}^{u}[j, k]\right\|^{2}\right\} .
$$

6.1. Performance of Sphere Decoder for MIMO-MBM. In this subsection, we present the number of add/multiply/comparison operations to evaluate performance of the proposed SD-MIMO-MBM developed in Section 3. Assume that the empirical parameter $\zeta=3$ [22] and the whole process is under time-invariant Rayleigh fading channels. We consider the case with $U=2$ transmit units, each transmit unit with 
TABLE 2: Simulation parameters.

\begin{tabular}{lc}
\hline Number of transmit units $U$ & 2 \\
Length of each frame $L$ & 100 \\
Number of frames $N_{f}$ & 10000 \\
Number of sinusoids to generate Rayleigh $W$ & 64 \\
One symbol duration in second $\triangle t$ & $0.5 \times 10^{-5} \mathrm{~s}$ \\
\hline
\end{tabular}

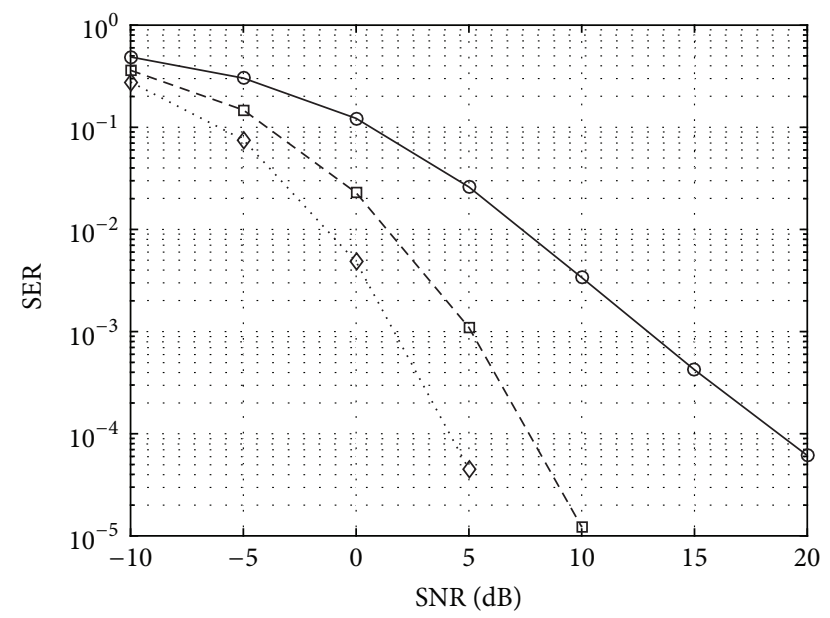

$$
\begin{array}{cl}
- & 2 \times 2 \text { MIMO-MBM with ML decoder } \\
--- & 2 \times 4 \text { MIMO-MBM with ML decoder } \\
\ldots . . . & 2 \times 6 \text { MIMO-MBM with ML decoder } \\
\circ & 2 \times 2 \text { MIMO-MBM with proposed SD } \\
\square & 2 \times 4 \text { MIMO-MBM with proposed SD } \\
\diamond \quad 2 \times 6 \text { MIMO-MBM with proposed SD }
\end{array}
$$

FIGURE 2: SER performances of the proposed sphere decoder and ML decoder for MIMO-MBM.

1 bit to be sent, and the number of receive antennas $N=$ $\{2,4,6\}$.

Figure 2 compares the SER performances of the proposed SD decoder and ML decoder for MIMO-MBM. We can readily see that SD-MIMO-MBM and ML decoder have an identical performance. Therefore, in the following, all the figures regarding the SER performance are plotted by using the SD-MIMO-MBM algorithm. The radio of computational complexity between the sphere decoder and the ML decoder, which is defined as

$$
\kappa=\frac{\psi_{\text {SD-MIMO-MBM }}}{\psi_{\mathrm{ML}}},
$$

is depicted in Figure 3. We can see that (1) a higher reduction in computational complexity is achieved for high SNR values for both decoders; (2) compared to traditional SD decoder, a higher reduction is achieved for more receive antennas, which means that the larger the constellation size is, the more reduction can be achieved for SD-MIMO-MBM; (3) the computation complexity of SD-MIMO-MBM is slightly higher than that of traditional SD for MIMO-MBM when the number of receive antennas is 2 due to the small constellation size. This is because the SD-MIMO-MBM decoder needs a few more comparison operations.

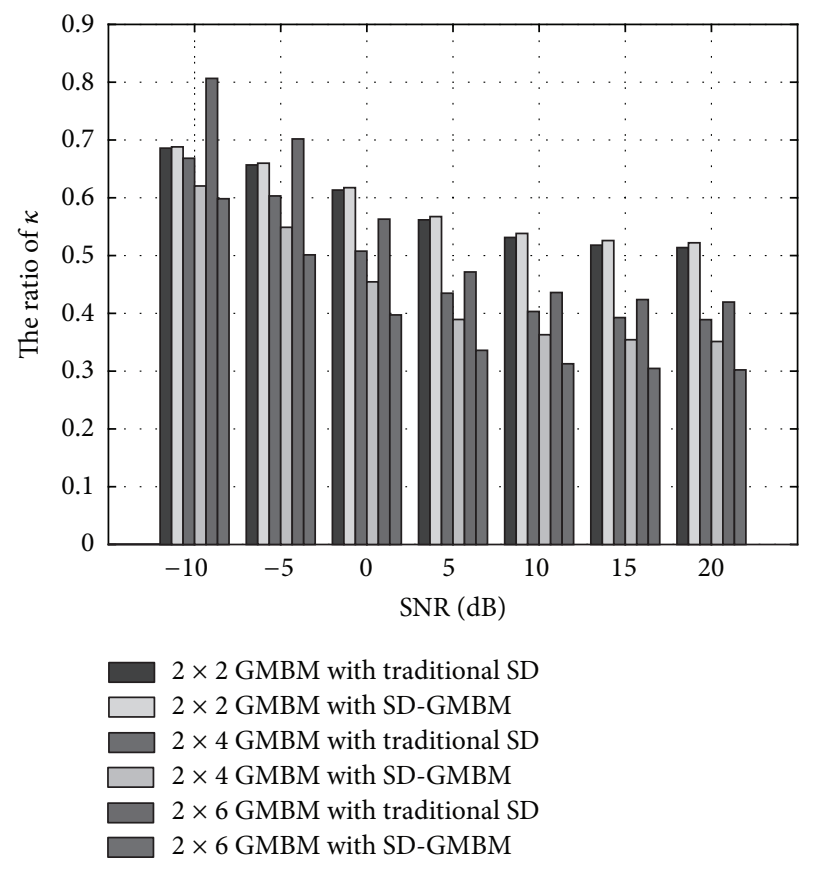

FIgURE 3: The calculation complexities of the proposed sphere decoder, the traditional sphere decoder, and the ML decoder for MIMO-MBM.

6.2. Performance of MIMO-MBM in Time-Selective Channels. In this subsection, we evaluate performances of MIMOMBM in both time-invariant channels and time-selective channels. We consider the case with $U=2$ transmit units, each transmit unit with 2 bits to be sent, and the number of receive antennas $N=\{2,4,6\}$. The receiver is assumed to have perfect knowledge of the channel matrix during the pilot estimation phase.

The average SER performance of MIMO-MBM versus SNR with different channels and different receive antennas is shown in Figure 4. As for time-selective channels, we assume that the maximum Doppler shift is $100 \mathrm{~Hz}$ and the maximum variation of channel factors $\alpha$ is 0.96 . We can see the following: (1) compared with the time-invariant channel case, the SER performance loss of MIMO-MBM in time-selective channels is negligible at low SNR; (2) the SER performance of time-selective channel saturates to a constant value in the high SNR region, which implies that higher SNR cannot improve the SER performance for time-selective channels; (3) as the number of receive antennas grows large, the SER performance can be significantly improved, since the constellation size also grows large, which results in improving the accuracy of transmission.

Figure 5 shows the average SER performance of MIMOMBM versus the maximum variation of channel factor $\alpha$ for time-invariant and time-selective channels with $N=4$ receive antennas and SNR $=10 \mathrm{~dB}$. The range of $\alpha$ is from 0.84 to 1 and the change of $\alpha$ is only based on the change of maximum Doppler shift. We can see that as $\alpha$ grows larger, the change of channel becomes slower and the SER performance goes better. When $\alpha$ is close to 1 , the SER performance of MIMO-MBM in time-selective channels is getting close to that in time-invariant channels. 


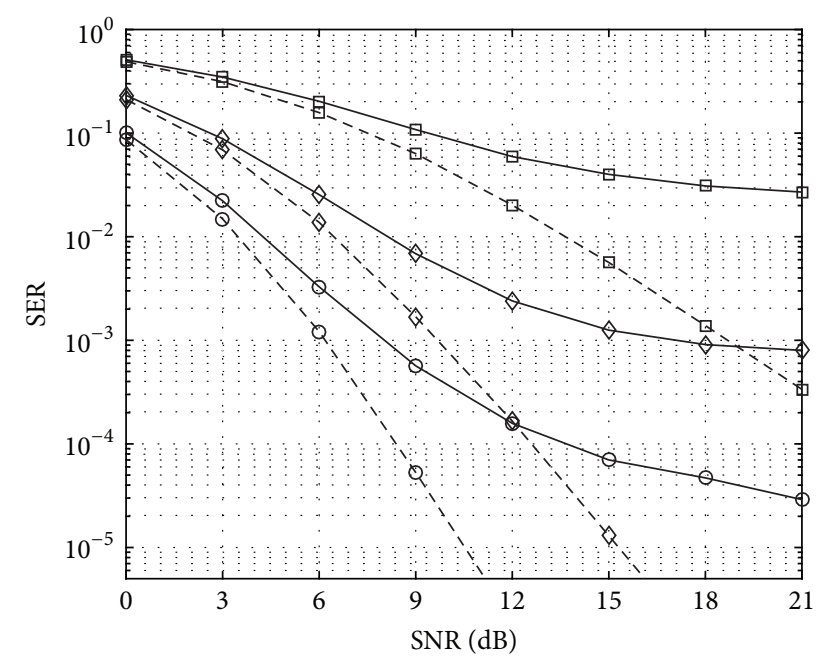

$\begin{array}{ll}\square & 2 \times 2 \text { MIMO-MBM time-selective } \\ -\Xi- & 2 \times 2 \text { MIMO-MBM time-invariant } \\ \diamond & 2 \times 4 \text { MIMO-MBM time-selective } \\ -\diamond- & 2 \times 4 \text { MIMO-MBM time-invariant } \\ - & 2 \times 6 \text { MIMO-MBM time-selective } \\ -\ominus- & 2 \times 6 \text { MIMO-MBM time-invariant }\end{array}$

FIGURE 4: SER performances of MIMO-MBM versus SNR for timeinvariant and time-selective channels (2 RF mirrors are used in each transmit unit).

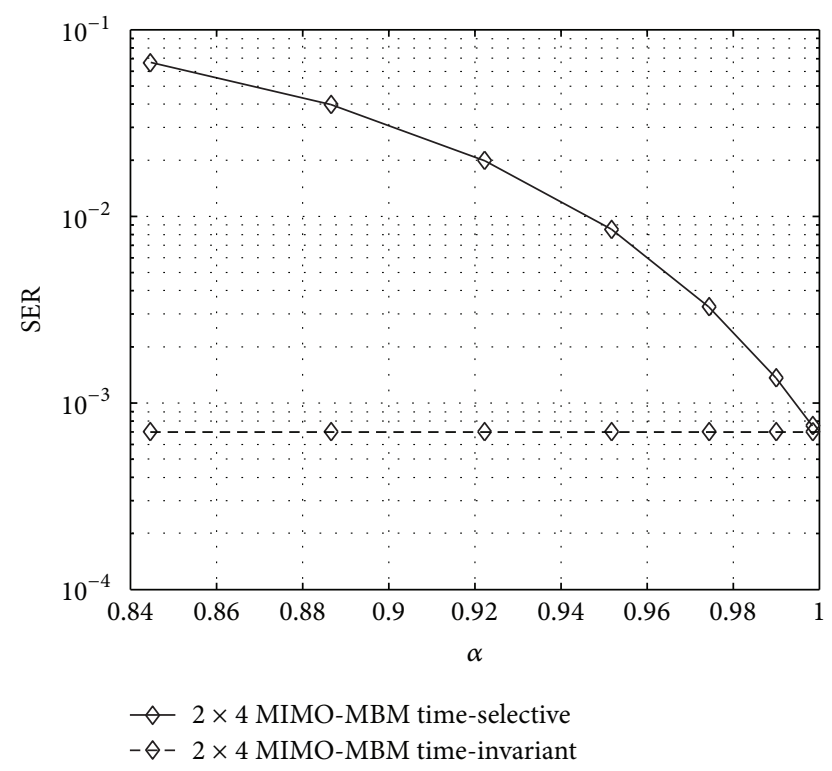

FIGURE 5: SER performance of MIMO-MBM versus the maximum variation of channel factor $\alpha$ for time-invariant and time-selective channels with $U=2, N=4$, and SNR $=10 \mathrm{~dB}$.

6.3. Performances of $L M S-M B M$ and RLS-MBM. In this subsection, we evaluate the performance of channel tracking algorithms discussed in Section 5 and the performances of MIMO-MBM in time-selective channels with LMS-MBM and RLS-MBM. For simplicity, we assume that the number of receive antennas $N=4$, the length of pilot sequences is $T_{t}=M$, the step size parameter $\mu$ of LMS-MBM is 0.1 , and the

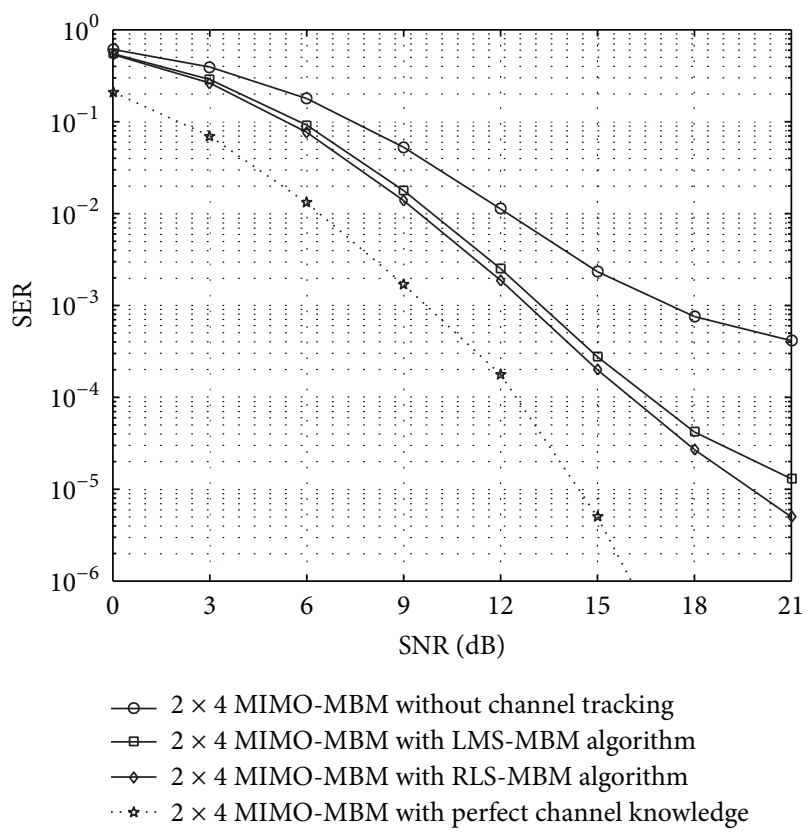

FIGURE 6: Average SER curves of $2 \times 4$ MIMO-MBM versus SNR in time-selective channels with and without LMS-MBM or RLS-MBM algorithms.

initial parameter $\delta$ and forgetting parameter $\lambda$ of RLS-MBM are 100 and 0.99 , respectively.

Figure 6 shows the average SER of MIMO-MBM versus the SNR over time-invariant and time-selective channels with and without channel tracking algorithms. We can see that the SER performance of MIMO-MBM in time-selective channels without channel tracking algorithm is the worst. The SER with LMS-MBM algorithm and RLS-MBM algorithm both outperform the SER without channel tracking algorithm. However, RLS-MBM performs better than the LMS-MBM. For example, when the target SER is $10^{-2}$, the SNR degradation of the one with LMS-MBM is $2.3 \mathrm{~dB}$ and the SNR degradation of the one with RLS-MBM is $2.7 \mathrm{~dB}$. But there is still room compared to the one in time-invariant channels because the SNR degradation is $5.8 \mathrm{~dB}$ compared to the one in time-selective channels without channel tracking.

The MSE of MIMO-MBM versus the SNR with different channel tracking algorithms is plotted in Figure 7. We can see that when the SNR is low, the curves of LMS-MBM and RLS-MBM are overlapped and both of them outperform pilot-aided estimation. When the SNR is high, RLS-MBM performs better than LMS-MBM. Figure 8 shows the MSE versus the maximum variation of channel factors $\alpha$ at $\mathrm{SNR}=$ $10 \mathrm{~dB}$. We can see that when $\alpha=1$, the channel becomes time-invariant. However, the MSEs of LMS-MBM and RLSMBM increase with the decreasing of $\alpha$, which implies that the faster the channel changes, the larger the estimation error is, even if the channel tracking algorithms are used. Figure 9 shows the SER versus the maximum variation of channel factors. We can see that when the target SER is $10^{-2}$, the coefficient $\alpha$ of LMS-MBM and RLS-MBM estimated channels can be about 0.959 and 0.885 , respectively. 


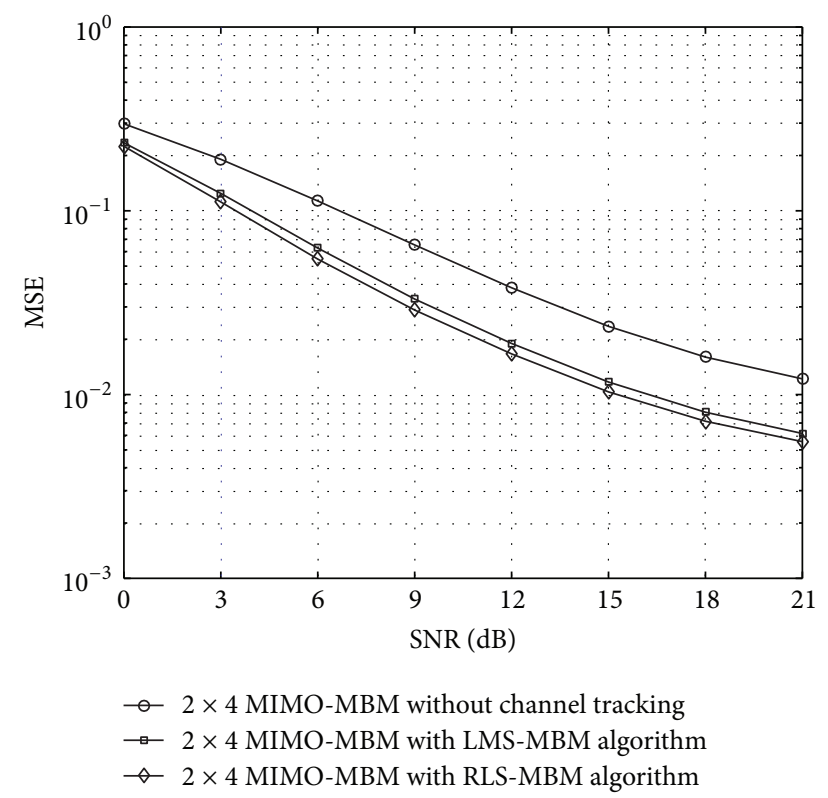

FIGURE 7: Average MSE performances of LMS-MBM and RLS-MBM algorithms versus SNR.

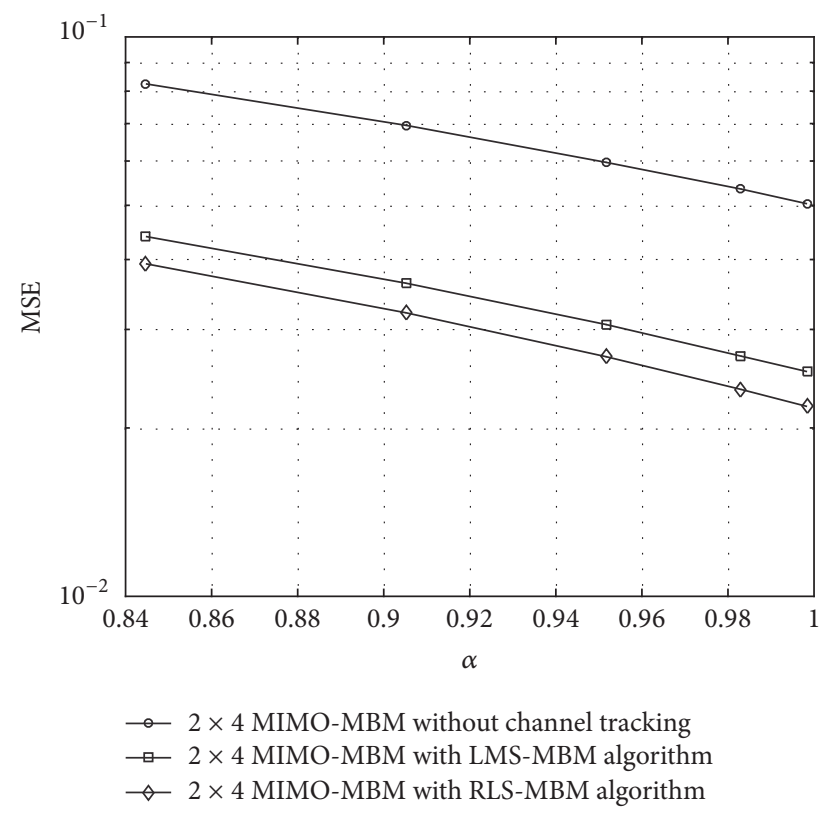

FIGURE 8: Average MSE performances of LMS-MBM and RLSMBM algorithms versus the maximum variation of channel factor $\alpha$.

LMS-MBM and RLS-MBM algorithms are only carried out once during the channel tracking process in Figures 6-9. Actually, system can improve the reliability by implementing channel tracking algorithms more times at each symbol. Furthermore, during the pilot-aided channel estimation, the length of pilots affects the accuracy of channel tracking. Apparently, better SER performance can be obtained if the length of pilots is long. However, since using longer pilots reduces the spectral efficiency of system, it is very important to choose the reasonable length of pilots. MBM system needs

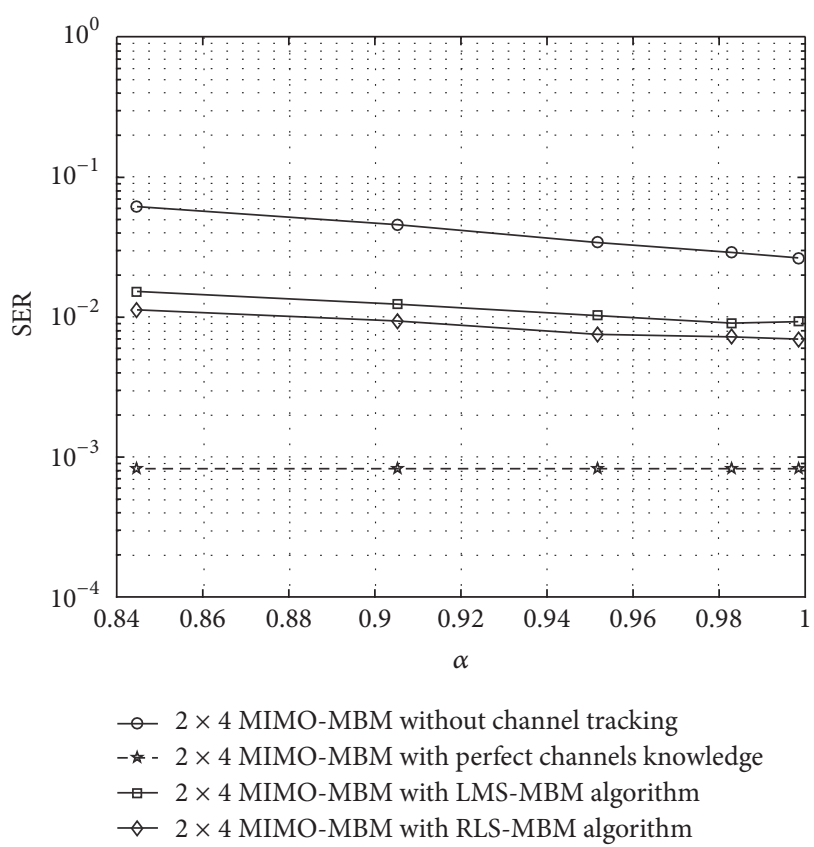

FIGURE 9: Average SER performances of LMS-MBM and RLS-MBM algorithms versus the maximum variation of channel factor $\alpha$.

to transmit pilots by scanning through all the channel states, which makes MBM need at least $M$ pilots.

Figure 10 gives the performance of LMS-MBM and RLSMBM with $T_{t}=\{M, 2 M, 3 M\}$ pilots, respectively. We can see that as the number of pilots gets larger, the performance of LMS-MBM and RLS-MBM gets better. As for LMS-MBM, when the target SER is $10^{-2}$, the corresponding SNRs for MBM with $M, 2 M$, and $3 M$ pilots are $9.9 \mathrm{~dB}, 8.5 \mathrm{~dB}$, and $8 \mathrm{~dB}$, respectively. As for RLS-MBM, when the target SER is $10^{-2}$, the corresponding SNRs for MBM with $M, 2 M$, and $3 M$ pilots are $9.5 \mathrm{~dB}, 8.2 \mathrm{~dB}$, and $7.8 \mathrm{~dB}$, respectively. Therefore, longer pilot training length results in a better performance of LMS-MBM and RLS-MBM.

\section{Conclusion}

In this paper, by employing the GSM-MBM model, a reduced complexity sphere decoder and two channel tracking algorithms called LMS-MBM and RLS-MBM in time-selective channels based on the MIMO-MBM model are proposed. The proposed sphere decoder performs as well as ML decoder in SER performance and reduces the complexity of the decoding process, especially when the constellation size is large. Two channel tracking algorithms based on least mean square adaptive filter and recursive least-squares adaptive filter called LMS-MBM and RLS-MBM are proposed. The complexities of these two algorithms are equal to each other on a macro level, but RLS-MBM is more complicated than LMS-MBM in some details. Numerical simulations verify that although the two channel tracking algorithms improve systems' SER performance significantly, RLS-MBM outperforms the LMS-MBM. Meanwhile, the length of pilots affects the performance of LMS-MBM and RLS-MBM. Longer pilot 

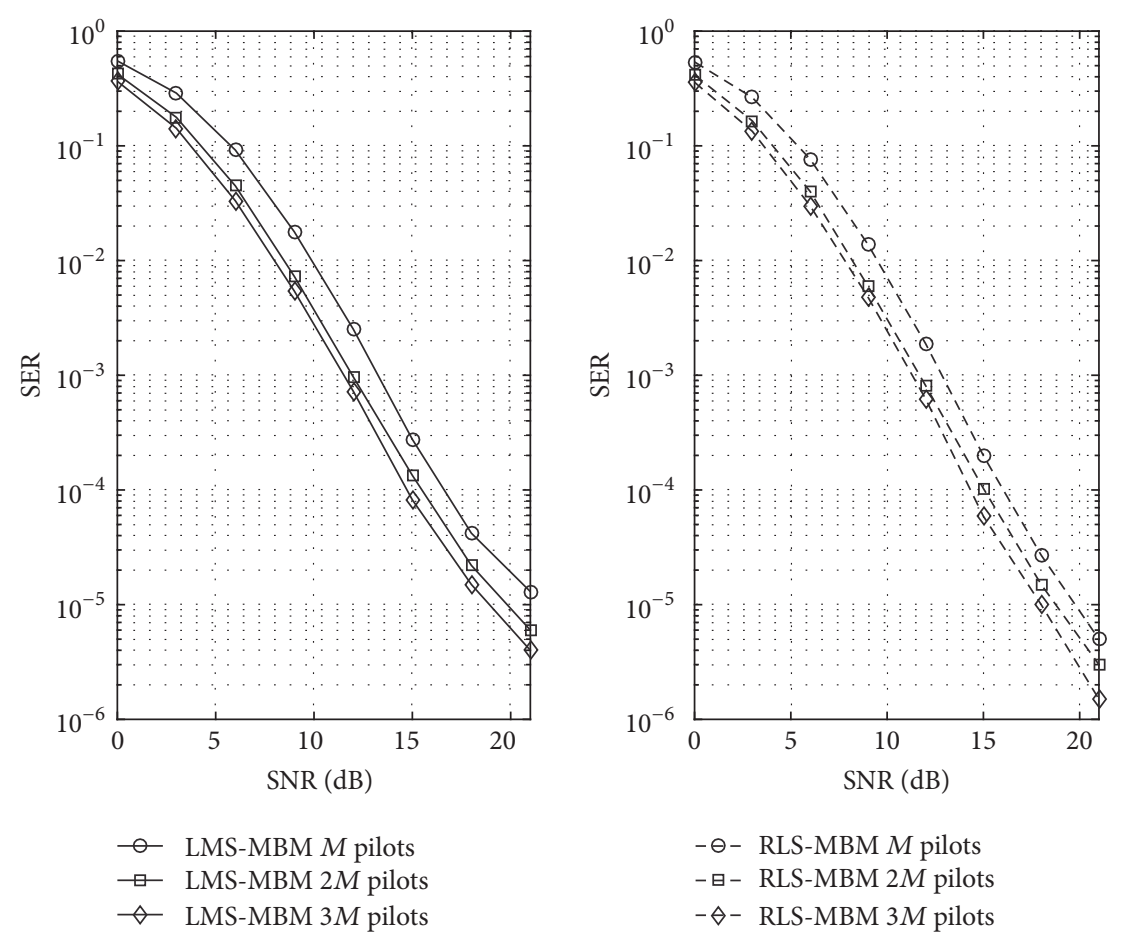

Figure 10: Performance of LMS-MBM and RLS-MBM with different length of pilots.

training length results in a better performance. The details about the performances of the proposed sphere decoder and two tracking algorithms were illustrated by simulations.

\section{Conflicts of Interest}

The authors declare that they have no conflicts of interest.

\section{Acknowledgments}

The research was supported in part by the NSFC Project (Grant no. 61471027), Fundamental Research Funds for the Central Universities (Grant 2017JBM306), Beijing Nova Programme (no. Z161100004916068), the Open Research Fund of National Mobile Communications Research Laboratory, Southeast University (Grant no. 2017D01), and Beijing Science and Technology Talents Program (2016023).

\section{References}

[1] A. K. Khandani, "Media-based modulation: a new approach to wireless transmission," in Proceedings of the 2013 IEEE International Symposium on Information Theory, ISIT 2013, pp. 3050-3054, July 2013.

[2] A. K. Khandani, "Media-based modulation: Converting static Rayleigh fading to AWGN," in Proceedings of the 2014 IEEE International Symposium on Information Theory, ISIT 2014, pp. 1549-1553, June 2014.

[3] K. Vamaraju, Single input multiple output media based modulation [Ph.D. thesis], University of Waterloo, 2015.
[4] E. Basar and I. Altunbas, "Space-time channel modulation," IEEE Transactions on Vehicular Technology, vol. 66, no. 8, pp. 7609-7614, 2017.

[5] E. Basar, "Index modulation techniques for $5 \mathrm{G}$ wireless networks," IEEE Communications Magazine, vol. 54, no. 7, pp. 168$175,2016$.

[6] S. Sanayei and A. Nosratinia, "Antenna selection in MIMO systems," IEEE Communications Magazine, vol. 42, no. 10, pp. 68-73, 2004.

[7] R. W. Heath Jr., S. Sandhu, and A. Paulraj, "Antenna selection for spatial multiplexing systems with linear receivers," IEEE Communications Letters, vol. 5, no. 4, pp. 142-144, 2001.

[8] C. G. Christodoulou, Y. Tawk, S. A. Lane, and S. R. Erwin, "Reconfigurable antennas for wireless and space applications," Proceedings of the IEEE, vol. 100, no. 7, pp. 2250-2261, 2012.

[9] J. Costantine, Y. Tawk, S. E. Barbin, and C. G. Christodoulou, "Reconfigurable antennas: design and applications," Proceedings of the IEEE, vol. 103, no. 3, pp. 424-437, 2015.

[10] J. Jeganathan, A. Ghrayeb, L. Szczecinski, and A. Ceron, "Space shift keying modulation for MIMO channels," IEEE Transactions on Wireless Communications, vol. 8, no. 7, pp. 3692-3703, 2009.

[11] C. Xu and M. Zhao, "Mutual information and secrecy enhancement analysis of media-based modulation," in Proceedings of the 8th International Conference on Wireless Communications and Signal Processing, WCSP 2016, p. 5, 1, October 2016.

[12] Y. Naresh and A. Chockalingam, "On media-based modulation using RF mirrors," IEEE Transactions on Vehicular Technology, vol. 66, no. 6, pp. 4967-4983, 2017.

[13] E. Viterbo and J. Boutros, "A universal lattice code decoder for fading channels," Institute of Electrical and Electronics Engineers. Transactions on Information Theory, vol. 45, no. 5, pp. 16391642, 1999. 
[14] T. Zhou, C. Tao, S. Salous, L. Liu, and Z. Tan, "Channel sounding for high-speed railway communication systems," IEEE Communications Magazine, vol. 53, no. 10, pp. 70-77, 2015.

[15] Y. R. Zheng and C. Xiao, "Improved models for the generation of multiple uncorrelated Rayleigh fading waveforms," IEEE Communications Letters, vol. 6, no. 6, pp. 256-258, 2002.

[16] Z. Liu, X. Ma, and G. B. Giannakis, "Space-time coding and Kalman filtering for time-selective fading channels," IEEE Transactions on Communications, vol. 50, no. 2, pp. 183-186, 2002.

[17] B. Hassibi and B. M. Hochwald, "How much training is needed in multiple-antenna wireless links?" IEEE Transactions on Information Theory, vol. 49, no. 4, pp. 951-963, 2003.

[18] R. Couillet and M. Debbah, Random Matrix Methods for Wireless Communications, Cambridge University Press, Cambridge, UK, 2011.

[19] D. G. Manolakis, V. K. Ingle, and S. M. Kogon, Statistical and Adaptive Signal Processing: Spectral Estimation, Signal Modeling, Adaptive Filtering, and Array Processing, McGraw-Hill, Boston, Mass, USA, 2008.

[20] K. A. K. Seifi E and M. Atamanesh, "Media-based MIMO: a new frontier in wireless communications," https://arxiv.org/pdf/ 1507.07516.pdf.

[21] Y. Li, "Simplified channel estimation for OFDM systems with multiple transmit antennas," IEEE Transactions on Wireless Communications, vol. 1, no. 1, pp. 67-75, 2002.

[22] O. Xia, H. Hu, and $\mathrm{H}$. Wang, "Reduced initial searching radius for sphere decoder," in Proceedings of the 18th Annual IEEE International Symposium on Personal, Indoor and Mobile Radio Communications, PIMRC'07, pp. 1-4, September 2007. 


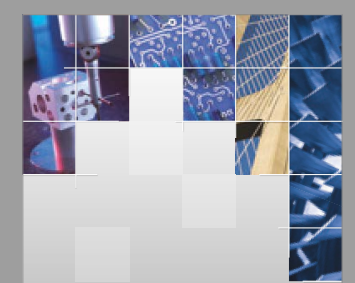

\section{Enfincering}
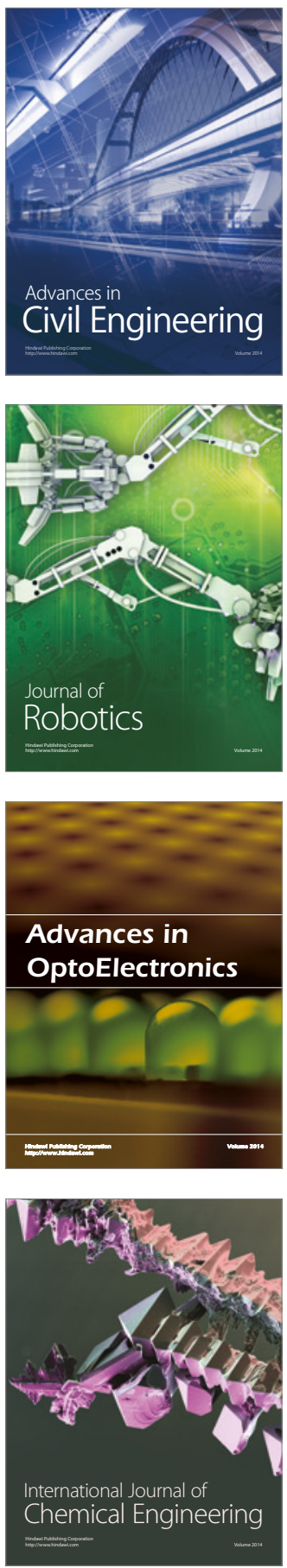

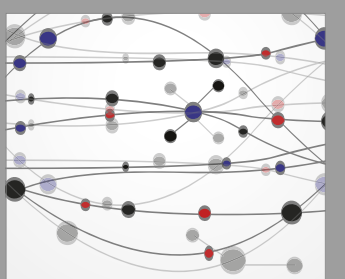

The Scientific World Journal

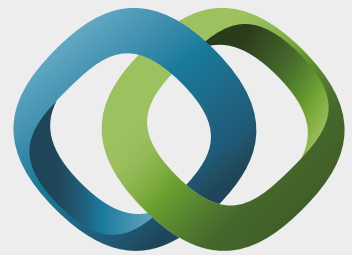

\section{Hindawi}

Submit your manuscripts at

https://www.hindawi.com
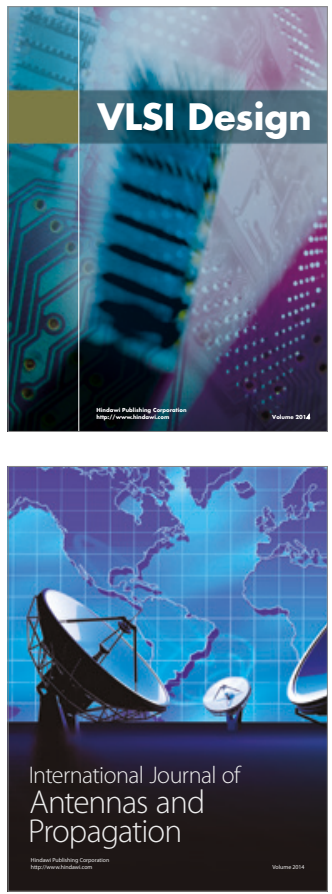

\section{Rotating}

Machinery
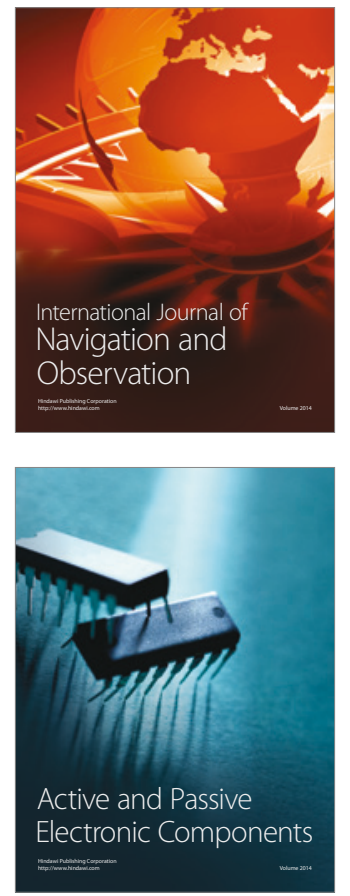
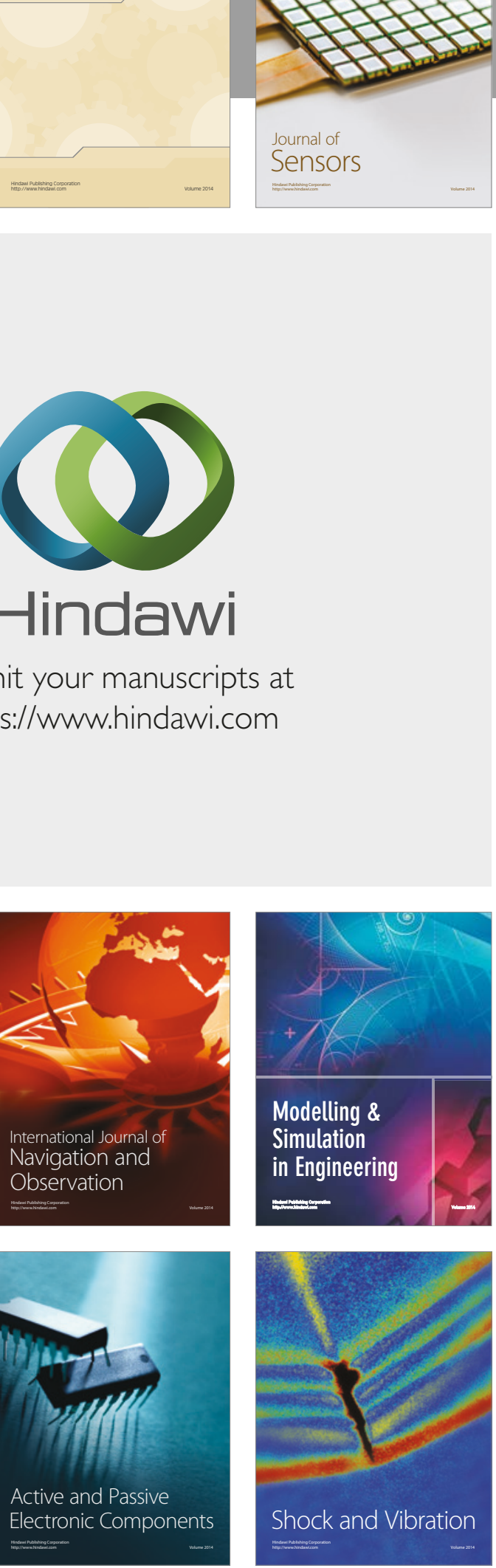
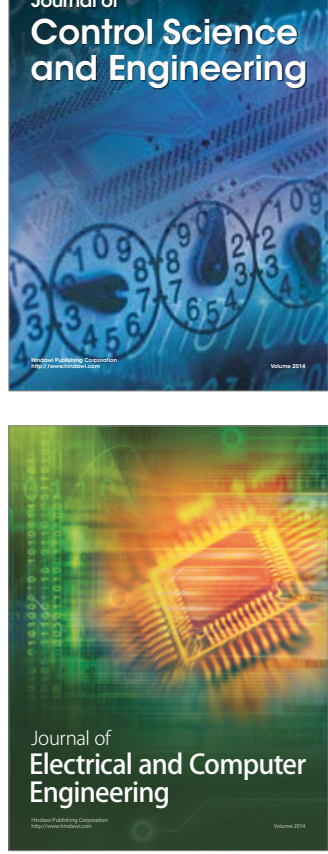

Distributed

Journal of

Control Science

and Engineering
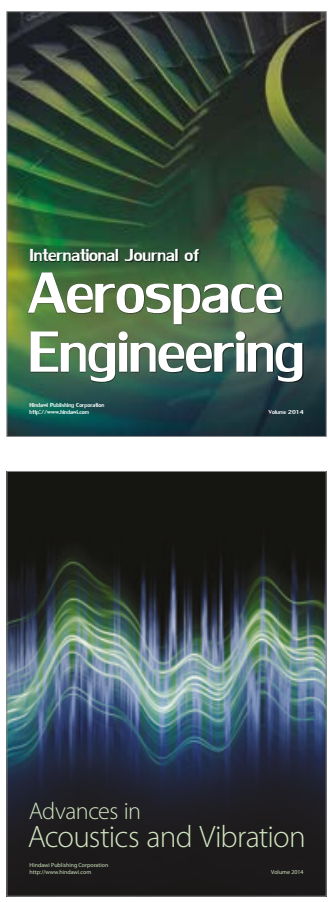

Sensor Networks 